\title{
A-scan biometry, phacoemulsification, and foldable intraocular lens implantation in a young orangutan (Pongo pygmaeus)
}

\author{
Natthanet Sritrakoon ${ }^{1}$, Winyu Karntip ${ }^{1}$, Sirirat Niyom², Susadee Khemton 3 , Panuwat Pakpiboon ${ }^{3}$, \\ Kant Wongsirodkul ${ }^{3}$, Kanyarat Arunsri ${ }^{1}$ and Aree Thayananuphat ${ }^{*}$ \\ ${ }^{1}$ Ophthalmology Unit, Kasetsart University Veterinary Teaching Hospital, Bangkok, Thailand \\ ${ }^{2}$ Department of Companion Animal Clinical Sciences, Faculty of Veterinary Medicine, Kasetsart University, \\ Bangkok, Thailand \\ ${ }^{3}$ Veterinary Department, Safari World, Bangkok, Thailand
}

\begin{abstract}
Background: Cataracts are the major cause of visual impairment in animals which can be curable by surgical treatment. Phacoemulsification is the standard technique for cataract treatment that is applied to almost all species with a high success rate.

Case Description: A 2-year-old intact female orangutan (Pongo pygmaeus) was presented for the study having bilateral opacity of the lenses, for 2 weeks. Ophthalmic examination revealed mature cataract OU. Ocular biometry measurements using A-scan ultrasonography for appropriate intraocular lens (IOL) refractive power calculation were carried out. Electroretinography was applied to ensure retinal function is intact. The orangutan underwent phacoemulsification OU and +24 diopter IOL implantation OS to restore vision. IOL implantation was not carried out OD because of a posterior capsular tear. Retinoscopy after 3 weeks postoperatively revealed +2.0 diopters OS. The outcome of the cataract surgery was successful during 3 years follow-up. The orangutan lived with other orangutans and was alert with normal behavior such as catching food, climbing trees, and swinging hand over hand from one branch to another.

Conclusion: Cataract surgery with phacoemulsification OU and adjusted IOL implantation OD was successful with few complications in this orangutan. Vision was restored with normal behavior, even though an adjusted IOL was inserted in only one eye.

Keywords: Biometry, cataract, orangutan, phacoemulsification, Pongo pygmaeus.
\end{abstract}

\section{Introduction}

Cataracts are opacities within the lens (Adkins and Hendrix, 2003). Cataracts can be caused by congenital, heredity, oxidative damage, nutritional deficiencies, senile changes, trauma, toxins, drugs, radiation therapy, inflammation, or previous metabolic diseases (Adkins and Hendrix, 2003; Montiani-Ferreira et al., 2010). Cataracts can be curable by surgical treatment. Phacoemulsification is the standard technique for cataract treatment that is applied to almost all species and has a high success rate (Lim et al., 2011; Leiva et al., 2012; Brooks et al., 2014; Sanchez et al., 2018; Fenollosa-Romero et al., 2020). There is a paucity of the published report of phacoemulsification in nonhuman primates, such as spider monkeys, gorillas, chimpanzees, pygmy marmosets, chacma baboons, and orangutans (Whitley et al., 1980; de Faber et al., 2004; Clouser, 2010; Montiani-Ferreira et al., 2010; Leiva et al., 2012; Warwick et al., 2017). Ocular biometry is a useful technique for the preoperative calculation of the intraocular lens (IOL) diopter power (Ademola-Popoola et al., 2015). However, some non-human primates have undergone surgery by phacoemulsification with IOL implantation without the IOL power calculation. To the authors' knowledge, there has been no published report of the preoperative calculation of the IOL dioptric power and the postoperative calculation of the refractive error in the orangutan.

This is the first report of A-scan biometry, preoperative calculation of the IOL dioptric power, and successful bilateral phacoemulsification with unilateral foldable IOL implantation in a young orangutan (Pongo pygmaeus).

\section{Case Details}

History and ophthalmic examination

A 2-year-old intact female orangutan from an open zoo in Bangkok was presented with bilateral opacity of the lenses, showing slow movement and bumping into objects during 2 weeks of observation. Ophthalmic examination revealed complete cataract OU. Menace response was negative, whereas dazzle reflex and pupillary light reflex were positive OU. The conjunctiva, cornea, anterior chamber, and iris were evaluated as normal using a slit lamp biomicroscope (Kowa SL17 Portable Slit-lamp biomicroscope, Kowa Co. Ltd, 
Tokyo, Japan). Intraocular pressures which were measured using a rebound tonometer (Icare ${ }^{\circledR}$ TonoVet, Icare Finland Oy, Helsinki, Finland) were normal (25 $\mathrm{mmHg}$ OU). Fundic examination could not be carried out due to cloudiness in the lenses OU. Definitive diagnosis was mature cataracts OU (Fig. 1). B-scan ocular ultrasonography (Logiq E9, GE Healthcare, WI) with topical $0.5 \%$ tetracaine hydrochloride (Alcon $^{\circledR}$, Alcon-Couvreur, Puurs, Belgium) revealed bilateral cataracts with no other ocular abnormalities (Fig. 2). Physical examination, hematology, and blood chemistry results were within normal limits. Cataract surgery with phacoemulsification and IOL implantation were planned. A-scan biometry was planned before the surgical date to prepare the IOL. Electroretinography was planned to be carried out just before phacoemulsification and dark adaptation was skipped to decrease the anesthetic period.

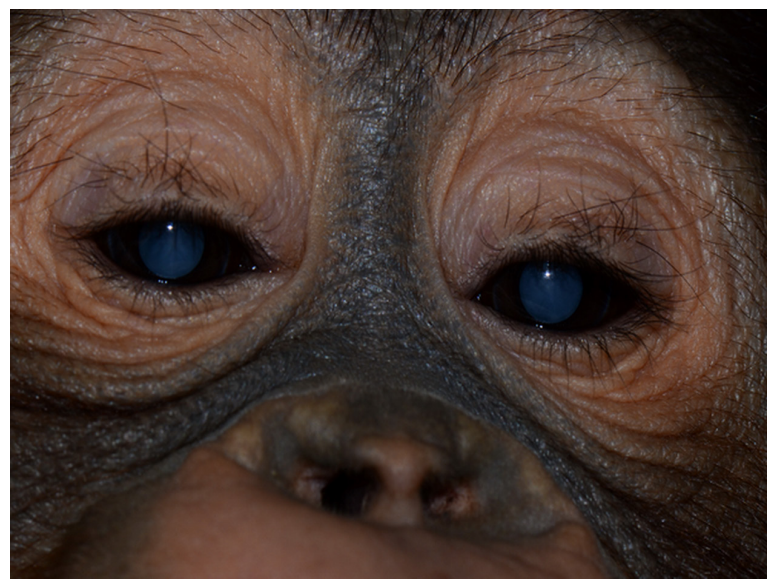

Fig. 1. Bilateral intumescent mature cataract causing visual loss in the orangutan.

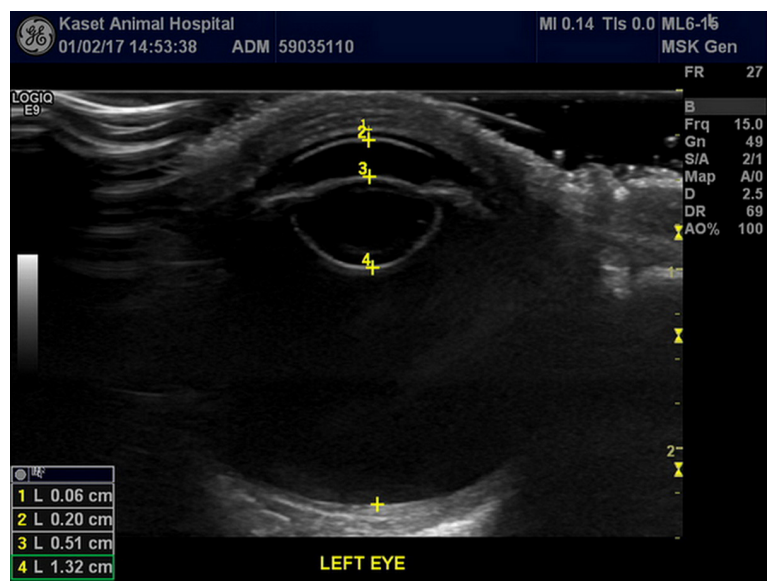

Fig. 2. B-scan ultrasonography revealing hyperechogenicity of lens capsule and cortex indicating cataract with normal other ocular structures for the left eye.

\section{A-scan biometry}

An immersion ultrasound A-scan biometry (Axis II PR, Quantel Medical, France) was carried out to determine the axial length (AL) and the IOL dioptric power calculation (Fig. 3). Anesthesia was carried out using intramuscular $2.5 \mathrm{mg} / \mathrm{kg}$ tiletamine-zolazepam (Zoletil, Virbac Laboratories, Carros, France), followed by topical anesthesia with $0.5 \%$ tetracaine hydrochloride (Alcon ${ }^{\circledR}$, Alcon-Couvreur, Puurs, Belgium). The scleral immersion shell, which was filled with normal saline, was placed on the cornea and positioned parallel to the axis of the eye. The average ALs were $21.54 \mathrm{~mm}$ OD and $21.22 \mathrm{~mm}$ OS (Fig. 4). The refractive powers of the IOLs were +25.48 diopter OD and +25.97 diopter OS and were calculated based on the SPK2 formula (Table 1). Since this orangutan was quite young, corneal flattening and increasing AL would be expected when growing up (Flitcroft et al., 1999). The chosen IOL dioptric power was $20 \%$ subtraction from the calculated dioptric power from the SPK-2 formula to avoid the development of high myopia. Therefore, IOLs with +23.5 diopter OD and +24 diopter OS were prepared for this surgery.

\section{Preoperative management and anesthesia}

Premedication was prescribed with topical 0.5\% moxifloxacin hydrochloride $\quad$ Vigamox $^{\circledR}$, Alcon

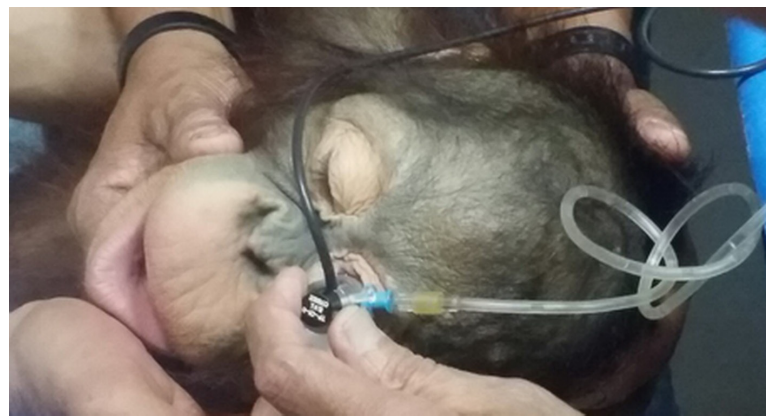

Fig. 3. The orangutan was under quick general anesthesia using tiletamine-zolazepam (Zoletil, Virbac Laboratories, Carros, France) to perform reliable A-scan biometry for IOL power calculation.

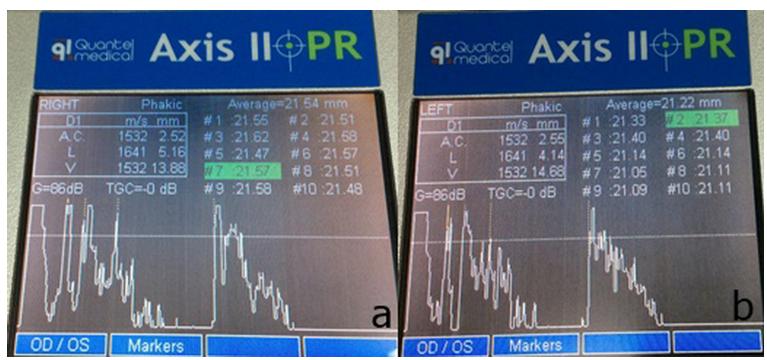

Fig. 4. A-scan biometry included anterior chamber (AC), Lens (L), vitreous (V), and average (AL) being displayed on the screen of the ultrasound both OD (a) and OS (b). 
Laboratories, Inc., Fort Worth, TX) and 1\% prednisolone acetate (Inf-Oph, Seng Thai Company, Bangkok, Thailand) four times a day for 3 days before surgery. One percent topical tropicamine (Mydriacyl ${ }^{\circledR}$, Alcon-Couvreur, Puurs, Belgium) was applied every 30 minutes for 2 hours before surgery. Intramuscular $1 \mathrm{mg} / \mathrm{kg}$ ketamine hydrochloride (Ketamine-Hameln, Siegfried Hameln GmbH, Hameln, Germany), 0.03 $\mathrm{mg} / \mathrm{kg}$ midazolam (Midazolam Sandoz ${ }^{\circledR}$, EVER Pharma Jena GmbH, Jena, Germany), and $0.01 \mathrm{mg} /$ $\mathrm{kg}$ atropine sulfate (Atropine, Atlantic Lab, Bangkok, Thailand) were used as pre-anesthesia. $0.7 \mathrm{mg} / \mathrm{kg}$ propofol (Troypofol $^{\mathrm{TM}}$, Troikaa Pharmaceuticals, Uttarakhand, India) was administered intravenously as an anesthetic induction. A $0.5 \mathrm{~cm}$ endotracheal tube (Tuoren, Henan Tuoren Medical Device, Henan, China) was intubated. Anesthesia was maintained with $2 \%$ isoflurane (Attane ${ }^{\mathrm{TM}}$, Piramal Critical Care, Inc., PA) inhalation. Intravenous acetar solution (R-CETATE, General Hospital Products Public Co., Ltd., Pathum Thani, Thailand) was administered during the anesthetic period. Local anesthesia at four sites of the peribulbar area with $4 \mathrm{mg}$ lidocaine hydrochloride (Locana, L.B.S. Laboratory, Bangkok, Thailand) was injected to stabilize the eyeball (Montiani-Ferreira et al., 2010).

\section{Electroretinography}

Electroretinography was carried out to access the retinal function using Handheld Multispecies electroretinography (HMsERG model 2000, Ocuscience LLC, MO) just before surgery. Dark adaptation was skipped to reduce the anesthetic period. A contact lens monopolar electrode (ERG-jet ${ }^{\circledR}$, Fabrinal

Table 1. A-scan biometry.

\begin{tabular}{lcc}
\hline Parameter & OD & OS \\
\hline Average of AL $(\mathrm{mm})$ & 21.54 & 21.22 \\
AL $(\mathrm{mm})$ & 21.57 & 21.37 \\
\hline ACD $(\mathrm{mm})$ & 2.52 & 2.55 \\
LT $(\mathrm{mm})$ & 5.16 & 4.14 \\
Vitreous depth $(\mathrm{mm})$ & 13.88 & 14.68 \\
SRK-2 IOL (diopter) & 25.48 & 25.97 \\
SRK-T IOL (diopter) & 26.36 & 27.06 \\
\hline
\end{tabular}

SA, LaChaux-de-Fonda, Switzerland) was placed on the corneal surface and contained a drop of sterile ophthalmic gel-carbomer (Lacryvisc ${ }^{\circledR}$, Alcon ${ }^{\circledR}$, AlconCouvreur, Puurs, Belgium). Two platinum subdermal needle electrodes (Viasys ${ }^{\mathrm{TM}}$ Healthcare, Neurocare group, Madison, WI) were placed at the occiput and at $3 \mathrm{~cm}$ from the eyelid margin rostral to the base of the ear to be used as a reference and ground electrodes, respectively. The result of the electroretinography based on the QuickRetCheck protocol is shown in Table 2 and Fig. 5. At standard light intensity $(3,000$ mcd.s $/ \mathrm{m}^{2}$ ) stimulation, the a-wave amplitudes were $101.3 \mu \mathrm{v}$ OD and $113.2 \mu \mathrm{v}$ OS and the implicit times were 18.8 mseconds OD and 18.6 mseconds OS. The b-wave amplitudes were $305.2 \mu \mathrm{v}$ OD and $442.1 \mu \mathrm{v}$ OS and the implicit times were 42.0 mseconds OD and 43.6 mseconds OS.

\section{Pre-surgical preparation and phacoemulsification technique}

Standard surgical preparation was carried out by eyelash clipping and conjunctival flushing with 1:50 povidoneiodine solution diluted. Intramuscular enrofloxacin (Baytril ${ }^{\circledR}$, Bayer Korea Ltd., Ansan, Korea) $2.5 \mathrm{mg} /$ $\mathrm{kg}$ and intravenous $0.2 \mathrm{mg} / \mathrm{kg}$ flunixin meglumine (Finadyne $^{\circledR}$, Schering-Plough Sante Animale, Segre, France) were administered. An incision site was made at 11 o'clock of the limbus using a $3.0 \mathrm{~mm}$ Slit Knife (Sharpoint ${ }^{\mathrm{TM}}$, Surgical Specialties Corporation, Tijuana, C.P., Mexico). Intracameral administration of $0.1 \mathrm{ml}$ of $0.1 \%$ trypan blue for the anterior lens capsule staining was carried out. The anterior chamber was filled with $2 \%$ sodium hyaluronate (Viscovet, AJL Ophthalmic, S.A., Álava, Spain) to stabilize the anterior chamber and protect the corneal endothelium. The anterior lens capsule was incised with a $25 \mathrm{~g}$ hypodermic needle and continuous capsulorhexis was conducted using Utrata capsulorhexis forceps. The anterior lens capsule was apparently soft and thin. Phacoemulsification using a one-handed technique was carried out in a phacoemulsification unit (Accurus, Alcon ${ }^{\circledR}$ Surgical, Keller, TX) at $70 \%$ phaco power. The lens material was soft. An IOL was not implanted OS due to posterior capsular rupture (1 $\mathrm{mm}$ diameter). An anterior vitrectomy was carried out OS. Cortical lens materials were completely removed using an I/A probe. Sodium hyaluronate was then aspirated from

Table 2. Electroretinography with QuickRetCheck protocol.

\begin{tabular}{cccccccccc}
\hline \multirow{2}{*}{$\begin{array}{c}\text { Flash } \\
\text { intensity } \\
\left(\mathbf{m} \mathbf{c d . s} / \mathbf{m}^{2}\right)\end{array}$} & \multicolumn{4}{c}{ O-wave } & \multicolumn{2}{c}{ b-wave } & \multicolumn{2}{c}{ a-wave } & \multicolumn{3}{c}{ OS-wave } \\
\cline { 2 - 10 } & $\begin{array}{c}\text { amplitude } \\
(\boldsymbol{\mu v})\end{array}$ & $\begin{array}{c}\text { implicit } \\
\text { time }(\mathbf{m S})\end{array}$ & $\begin{array}{c}\text { amplitude } \\
(\boldsymbol{\mu v})\end{array}$ & $\begin{array}{c}\text { implicit } \\
\text { time }(\mathbf{m S})\end{array}$ & $\begin{array}{c}\text { amplitude } \\
(\boldsymbol{\mu v})\end{array}$ & $\begin{array}{c}\text { implicit } \\
\text { time }(\mathbf{m S})\end{array}$ & $\begin{array}{c}\text { amplitude } \\
(\boldsymbol{\mu v})\end{array}$ & $\begin{array}{c}\text { implicit } \\
\text { time }(\mathbf{m S})\end{array}$ \\
\hline 10 & 6.5 & 29.9 & 40.2 & 95.9 & 7.1 & 43.2 & 55.9 & 94.8 \\
3,000 & 101.3 & 18.8 & 305.2 & 42.0 & 113.2 & 18.6 & 442.1 & 43.6 \\
10,000 & 110.4 & 19.0 & 419.2 & 44.1 & 208.5 & 18.1 & 517.8 & 47.1 \\
\hline
\end{tabular}




\begin{tabular}{|c|c|c|c|}
\hline 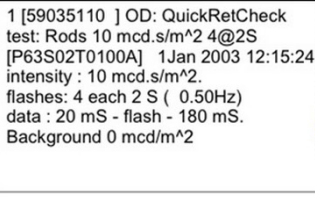 & $\begin{array}{l}\text {-- Waveform Data -- } \\
\text { a-wave } 6.5 \mathrm{uV} \text { at } 29.9 \mathrm{mS} \\
\text { b-wave } 40.2 \mathrm{uV} \text { at } 95.9 \mathrm{mS} \\
\text {--auto a/b } \\
\begin{array}{l}\text { Filtering: (4) } \\
\end{array}\end{array}$ & 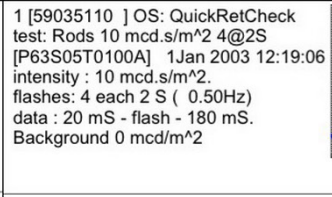 & $\begin{array}{l}\text {-- Waveform Data -- } \\
\text { a-wave } 7.1 \mathrm{uV} \text { at } 43.2 \mathrm{mS} \\
\text { b-wave } 55.9 \mathrm{uV} \text { at } 94.8 \mathrm{mS} \\
\text {--auto a/b } \\
\text { Filtering: (4) } \\
\end{array}$ \\
\hline 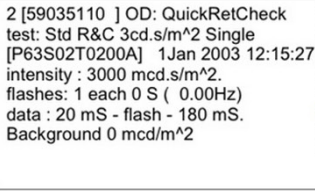 & $\begin{array}{l}\text { - Waveform Data -- } \\
\text { a-wave } 101.3 \mathrm{uV} \text { at } 18.8 \mathrm{mS} \\
\text { b-wave } 305.2 \mathrm{uV} \text { at } 42.0 \mathrm{mS} \\
\text {--auto a/b } \\
\text { Filtering: } \\
\quad \text { a2 }\end{array}$ & 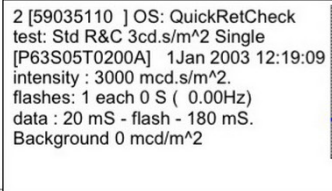 & $\begin{array}{l}\text {-- Waveform Data -- } \\
\text { a-wave } 113.2 \mathrm{uV} \text { at } 18.6 \mathrm{mS} \\
\text { b-wave } 442.1 \mathrm{uV} \text { at } 43.6 \mathrm{mS} \\
\text {--auto a/b } \\
\text { Filtering: } \quad \text { b2 }\end{array}$ \\
\hline 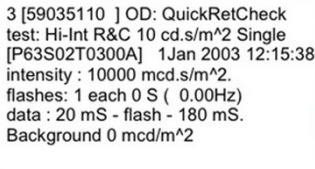 & 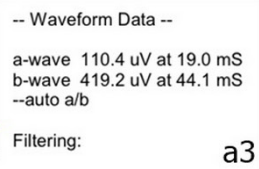 & 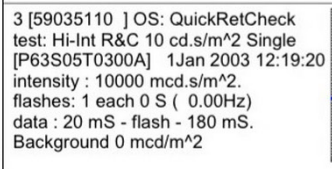 & $\begin{array}{l}\text { - Waveform Data -- } \\
\text { a-wave } 208.5 \mathrm{uV} \text { at } 18.1 \mathrm{mS} \\
\text { b-wave } 517.8 \mathrm{uV} \text { at } 47.1 \mathrm{mS} \\
\text {--auto a/b } \\
\text { Filtering: } \quad \text { b3 }\end{array}$ \\
\hline
\end{tabular}

Fig. 5. Result of electroretinography with QuickRetCheck protocol OD (a) OS (b); at low intensity responses $\left(10 \mathrm{mcd} . \mathrm{s} / \mathrm{m}^{2}\right)(\mathrm{a} 1$ and b1), standard intensity responses $\left(3,000 \mathrm{mcd} . \mathrm{s} / \mathrm{m}^{2}\right)\left(\mathrm{a} 2\right.$ and b2), and higher intensity responses $\left(10,000 \mathrm{mcd} . \mathrm{s} / \mathrm{m}^{2}\right)(\mathrm{a} 3 \mathrm{and} \mathrm{b3})$.
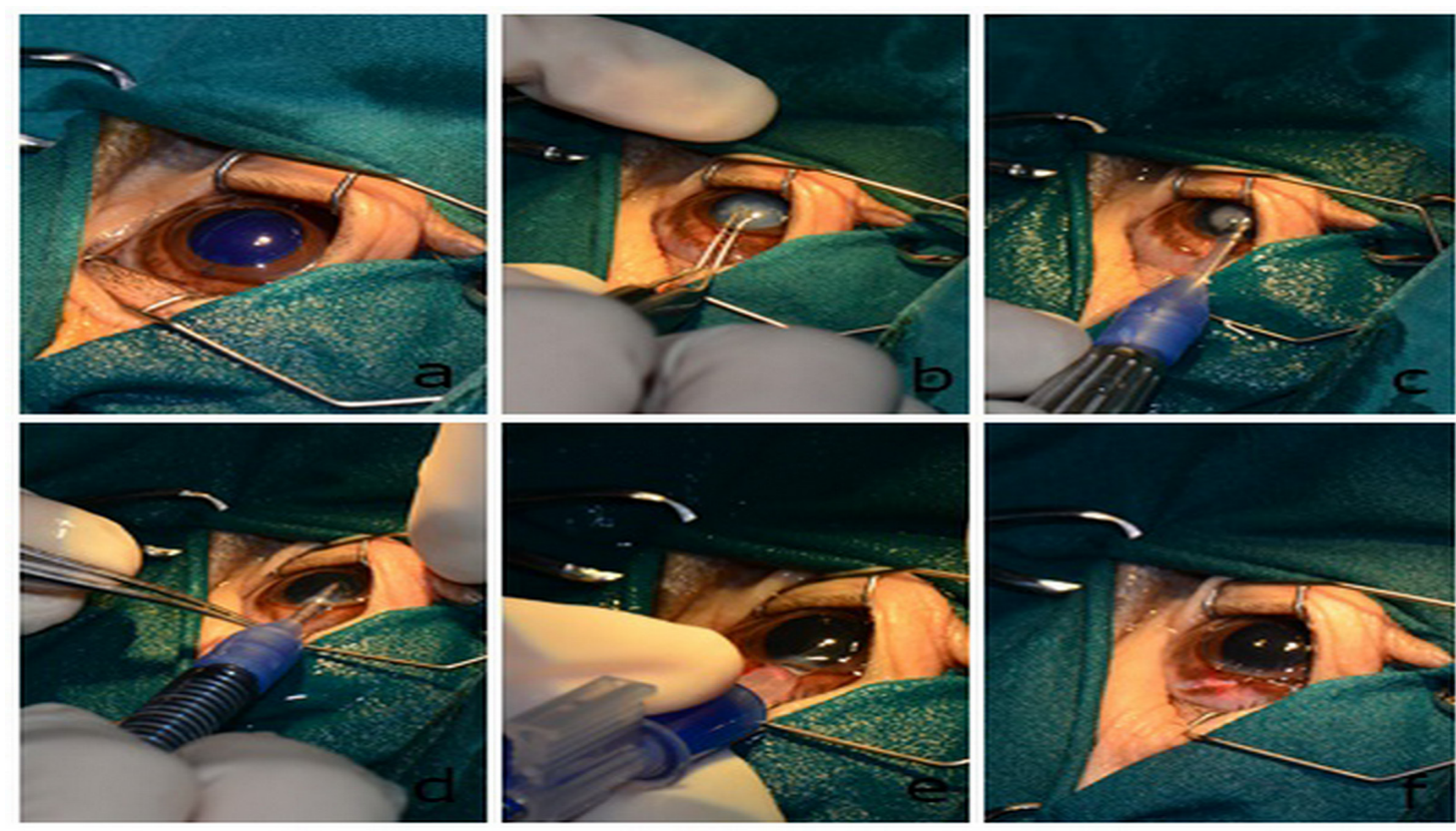

Fig. 6. Selected steps of the one-handed technique of phacoemulsification and IOL implantation OD. Anterior lens capsule was stained with trypan blue (a). Utrata capsulorhexis forceps were used to accomplish continuous curvilinear capsulorhexis with 6-8 $\mathrm{mm}$ diameter (b). One-handed phacoemulsification and aspiration technique were carried out (c). Irrigation and aspiration were then applied to remove all lens fibers (d). A +23.5 diopter IOL was inserted through the limbus incision to restore proper vision (e). The surgical wound at 11 o'clock was sutured with polyglycolic acid 9/0 (f).

the anterior chamber and capsular bag. The surgical wound was sutured using a simple, interrupted pattern with 9/0 polyglycolic acid suture material (PGA, FSSB Chirurgischenadeln GMBH, Jestetten, Germany). The balanced salt solution was injected to reform the anterior chamber. The same procedure was carried out in the contralateral eye, except an IOL (CT ASPHINA 509MP +23.5 diopter, Carl Zeiss, Dublin, CA) was inserted via a lens inserter (BLUEMIXS ${ }^{\mathrm{TM}} 180$ injector, Carl Zeiss,
Dublin, CA) OD (Fig. 6). The orangutan recovered from anesthesia uneventfully. Postoperative management with topical $0.5 \%$ moxifloxacin hydrochloride and $1 \%$ prednisolone acetate four times a day was administered for 3 weeks.

\section{Surgical outcome}

One day after surgery, the vision of the orangutan was restored. The orangutan could precisely catch the fruit that was thrown to her and quickly escaped all the 


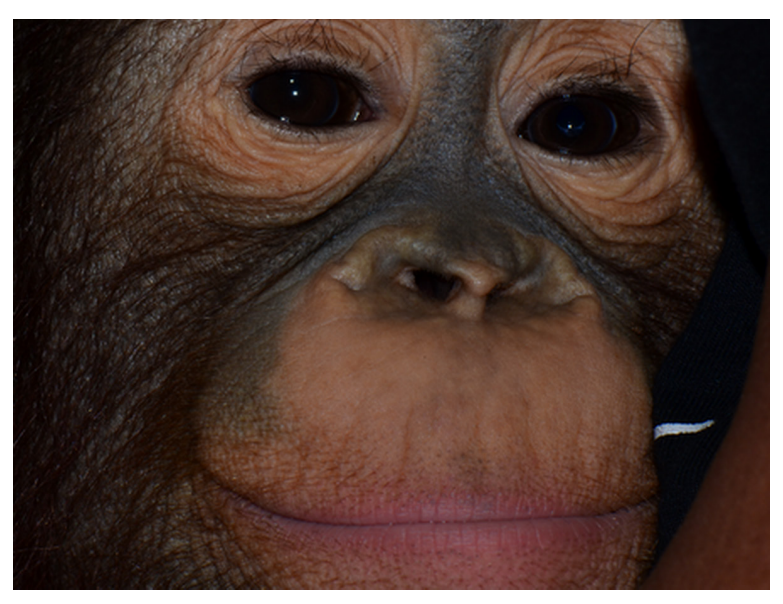

Fig. 7. Both eyes opened comfortably at 3 weeks postoperatively. Successful restoration of vision was tested by menace response and normal daily behavior.

obstacles to reach her caretaker. Neuroophthalmic tests (menace response, dazzle reflex, and pupillary light reflex) were positive OU. The IOP was $17 \mathrm{mmHg}$ OD and $20 \mathrm{mmHg}$ OS. Intramuscular prednisolone acetate $0.25 \mathrm{mg} / \mathrm{kg}$ (Prednisolone, T.P. drug Laboratories, Bangkok, Thailand) and $20 \mathrm{mg} / \mathrm{kg}$ amoxycillinclavulonic acid (Synulox ${ }^{\circledR}$, Haupt PharmaS.r.l, Latina, Italy) were prescribed for 1 week. The orangutan showed no adverse behavior to disturb the surgical wounds and consequently, an Elizabethan collar, eye patch, or other eye protection was unnecessary.

One week postoperatively, the presence of fibrin on the anterior lens capsule OD was found. Intramuscular prednisolone acetate $0.125 \mathrm{mg} / \mathrm{kg}$ was administered for 1 week. At 3 weeks postoperatively, no trace of fibrin was found in either of the eyes (Fig. 7). All ocular media remained clear. Topical $1 \%$ prednisolone acetate three times a day was administered for 2 more weeks and reduced to twice a day for 1 month before all medications were discontinued. Retinoscopy revealed hyperopia of +2.0 diopters OD. One month postoperatively, the orangutan was alert and exhibiting normal behavior such as grasping food or swinging hand over hand from one branch to another. The orangutan had a steady functional vision and all ocular media remained clear at 3 years follow-up. Postoperative complication presented as mild uveitis only for the first week after surgery.

\section{Discussion}

This is the first report of A-scan biometry, preoperative calculation of the IOL dioptric power, phacoemulsification with foldable IOL implantation, and postoperative calculation of the refractive error in the eye with an IOL in a young orangutan (Pongo pygmaeus). Generally, genetic origin has been suspected to be the cause of cataracts in captive primates (de Villiers et al., 2001), whereas congenital cataracts are the most common cause of treatable childhood blindness in children (Al Shamrani and Al Turkmani, 2012). Due to the young age of this orangutan with no history of ocular abnormalities, the cause of the cataracts was possibly congenital or inherited. Because of the concern, the cataract could be due to hereditary; this orangutan should not be allowed to breed because her offspring may have an unacceptably high probability of developing a cataract. Nutritional deficiencies have been considered as the cause of cataracts. However, this orangutan regularly received appropriate feed which contained cow milk, bread, hard-boiled egg, and a variety of fruits such as banana, papaya, pineapple, and orange. Therefore, a nutritional deficiency was less likely to be the cause of cataract in this orangutan.

A-scan ultrasound biometry was useful for intraocular structure measurements, such as the $\mathrm{AL}$, anterior chamber depth (ACD), and lens thickness (LT), that are used to calculate the power of intraocular lenses (Ademola-Popoola et al., 2015). AL in children can be measured using either a contact or immersion technique (Wilson and Trivedi, 2012). The immersion A-scan technique that was used in this case was more precise and has been recommended for pediatric IOL power calculation because the AL measured by contact A-scan was shorter than the actual AL in the eyes of pediatric patients (Wilson and Trivedi, 2012).

In general, uncooperative patients can result in inaccurate measurements for ocular biometry. The most important error in calculating the IOL dioptric power usually results from AL measurement (Wilson and Trivedi, 2012). To avoid such errors in uncooperative patients, ocular biometry, especially for AL should be carried out under general anesthesia to facilitate precise measurement (Wilson and Trivedi, 2012). The AL increases according to age (Flitcroft et al., 1999; Eibschitz-Tsimhoni et al., 2007) because adults have more corneal flattening than children. The selection of the IOL power in a growing eye is considered a major challenge. To avoid the development of increasing myopia with axial growth, $20 \%$ of the IOL power should be subtracted after the IOL calculation in young children (Yorston, 2001) to achieve emmetropia in the juvenile eye. Therefore, an IOL with a dioptric strength greater than the calculation was chosen for the young orangutan in the current study. After surgery, this orangutan would have slight hyperopia in the eye with the IOL. This condition should improve with age. In humans, optical correction or contact lenses are prescribed for residual hyperopia. However, these techniques are difficult to apply and might be unnecessary in animals. There are several IOL calculation formulas such as SPK/T, Holladay 1, Holladay 2, Hoffer Q, and Haigis. In children, the SRK/T and the Holladay 2 formulas provide the least prediction error in children younger than 2 years as compared to Holladay 1 and Hoffer Q (Vasavada et al., 2016). Therefore, the SPK-2 formula was used to calculate the IOL power in the current study. 
In animals under cataract extraction without IOL implantation, aphakic and hyperopia can be present (McMullen and Utter, 2010). A report stated that an aphakic orangutan was functionally competent at 3 months after surgery (Montiani-Ferreira et al., 2010). In general, an aphakic condition causes marked hyperopia which for an orangutan with the bilateral aphakic condition might need more time for adaptation. However, the orangutan in this report showed regained vision with precise grasping of objects within 1 day post-surgery.

Retinoscopy after phacoemulsification was carried out for the first time in this orangutan to evaluate the quality of vision. The postoperative refractive error of the eye with the IOL was +2.0 diopters which would be appropriate because the orangutan was able to quickly and precisely grasp fruits and climb trees on the day after surgery. As the orangutan grows up, the refractive error would be compensated and become emmetropic. Retinoscopy was not carried out in the aphakic left eye as an aphakic eye has a total loss of accommodation. Therefore, a high level of hyperopia would be expected (Sabiston, 1975). In this condition, the orangutan in the current study could compensate by using the eye with the IOL as the dominant eye for better vision (Sabiston, 1975).

Even though the dark adaptation was not undertaken before carrying out ERG, the a-wave amplitudes and implicit times could be obtained from both eyes when stimulated with low light intensity $\left(10 \mathrm{mcd} . \mathrm{s} / \mathrm{m}^{2}\right)$ compared to that reported using the same technique (Montiani-Ferreira et al., 2010). All a- and b-wave amplitudes seemed to be higher and all $\mathrm{a}$ and $\mathrm{b}$ implicit times seemed to be longer than previously reported in a 14-year-old female orangutan under a condition of 20-minutes dark adaptation (Montiani-Ferreira et al., 2010). This might have been due to the different ages of the animals. Normally, the amplitudes of ERG in young animals are higher than in aged animals because the number of photoreceptors and retinal pigment epithelial cells usually decrease with age (Dorey et al., 1989).

There was a 1-mm posterior capsular rent OD. In general, the dispersive viscoelastic solution could be injected to push the posterior capsule back prior to IOL insertion (Albou-Ganem and Barale, 2013). However, the lens capsule of this young orangutan was very soft and thin; consequently, there was no attempt to insert the IOL to prevent further tearing. To prevent capsular tearing in the future, the phaco tip should stay at the anterior center and the groove of the lens should not be made too deep by always observing red reflex during phacoemulsification.

Postoperative complications after cataract surgery in orangutan are generally not severe. In the current study, only mild anterior uveitis was observed at 1 week after the operation in this orangutan. No ocular inflammation was evident at 3 weeks postoperatively.
This corresponded to a previous report in which ocular inflammation was observed only within the first week after the operation (Montiani-Ferreira et al., 2010). Others postoperative complications in children have been glaucoma and rhegmatogenous retinal detachment (Fan et al., 2006) which were not evident in this orangutan. This report also confirmed that the orangutan was very tolerant of the surgical wound. No wound protection was necessary postoperatively after phacoemulsification. However, long-term PCO should be investigated thereafter.

In conclusion, cataract surgery with phacoemulsification OU and adjusted IOL implantation OD were successful with few complications in this orangutan. The vision was restored with normal behavior, even though an adjusted IOL was inserted in only one eye.

\section{Conflict of interest}

The authors declare that there is no conflict of interest.

\section{References}

Ademola-Popoola, D.S., Nzeh, D.A., Saka, S.E., Olokoba, L.B. and Obajolowo, T.S. 2015. Comparison of ocular biometry measurements by applanation and immersion A-scan techniques. J. Curr. Ophthalmol. 27, 110-114.

Adkins, E.A. and Hendrix, D.V.H. 2003. Cataract evaluation and treatment in dogs. Compendium. 25(11), 812-825.

Al Shamrani, M. and Al Turkmani, S. 2012. Update of intraocular lens implantation in children. Saudi. J. Ophthalmol. 26, 271-275.

Albou-Ganem, C. and Barale, P. 2013. Management of posterior capsular tear during phacoemulsification. Highl. Ophthalmol. 41: 11-14.

Brooks, D.E., Plummer, C.E., Carastro, S.M. and Utter, M.E. 2014. Visual outcomes of phacoemulsification cataract surgery in horses: 1990-2013. Vet. Ophthalmol. 17, 117-128.

Clouser, S. 2010. Cataract surgery with intraocular lens implantation on the captive western lowlands gorilla. Insight. 35, 13-16.

de Faber, J.T., Pameijer, J.H. and Schaftenaar, W. 2004. Cataract surgery with foldable intraocular lens implants in captive lowland gorillas (Gorilla gorilla gorilla). J. Zoo. Wildl. Med. 35, 520-524.

de Villiers, C., Seier, J.V. and Dhansay, M.A. 2001. Probable genetic origin for a large number of cataracts among captive-bred vervet monkeys (Chlorocebusaethiops). Am. J. Primatol. 55, 43-48.

Dorey, C.K., Wu, G., Ebenstein, D., Garsd, A. and Weiter, J.J. 1989. Cell loss in the aging retina. Invest. Ophthalmol. Vis. Sci. 30(8), 1691-1699.

Eibschitz-Tsimhoni, M., Archer, S.M. and Del Monte, M.A. 2007. Intraocular lens power calculation in children. Surv. Ophthalmol. 52, 474-482.

Fan, S.P.D., Yip, W.K., Christopher, B.Y., Rao, S.K. and Lam, D.S.C. 2006. Updates on the surgical management of pediatric cataract with primary 
intraocular lens implantation. Annal. Acad. Med. $35,564-570$.

Fenollosa-Romero, E., Jeanes, E., Freitas, I., Enache, A.E., Lockhart, R., Fleming, L., Knott, T.N.L., Dawson, C., Smith, K. and Busse, C. 2020. Outcome of phacoemulsification in 71 cats: a multicenter retrospective study (2006-2017). Vet. Ophthalmol. 23, 141-147.

Flitcroft, D.I., Knight-Nanan, D., Bowell, R., Lanigan, B. and O'Keefe, M. 1999. Intraocular lenses in children: changes in axial length, corneal curvature, and refraction. Br. J. Ophthalmol. 83, 265-269.

Leiva, M., Peña, T., Bayon, A., de León, M. and Morales, I. 2012. Phacoemulsification considerations in nonhuman primates. J. Med. Primatol. 41, 317-324.

Lim, C.C., Bakker, S.C., Waldner, C.L., Sandmeyer, L.S. and Grahn, B.H. 2011. Cataracts in 44 dogs (77 eyes): a comparison of outcomes for no treatment, topical medical management, or phacoemulsification with intraocular lens implantation. Can. Vet. J. 52, 283-288.

McMullen, R.J. Jr. and Utter, M.E. 2010. Current developments in equine cataract surgery. Equine. Vet. J. 37, 38-45.

Montiani-Ferreira, F., Lima, L., Bacellar, M., D’Otaviano Vilani, R.G., Fedullo, J.D. and Lange, R.R. 2010. Bilateral phacoemulsification in an orangutan (Pongopygmaeus).Vet. Ophthalmol. 13, 91-99.
Sabiston, D.W. 1975. Uniocular aphakia. Aust. N. Z. J. Ophthalmol. 3(1), 47-50.

Sanchez, R.F., Everson, R., Hedley, J., Dawson, C., Lam, R., Priestnall, S.L., Garcia de Carellan, A., de Miguel, C. and Seymour, C. 2018. Rabbits with naturally occurring cataracts referred for phacoemulsification and intraocular lens implantation: a preliminary study of 12 cases. Vet. Ophthalmol. 21(4), 399-412.

Vasavada, V., Shah, S.K., Vasavada, V.A., Vasavada, A.R., Trivedi, R.H., Srivastava, S. and Vasavada, S.A. 2016. Comparison of IOL power calculation formulae for pediatric eyes. Eye. 30, 1241-1250.

Warwick, A., Redrobe, S., Lotery, A. and Watts, J. 2017. Bilateral cataract surgery with intraocular lens implant in a captive western lowland gorilla. J. Med. Primatol. 46, 252-255.

Whitley, R.D., Jacobson, E.R., Lavach, J.D., Gelatt, K.N. and Barrie, K.P. 1980. Bilateral ultrasonic phacofragmentation and aspiration cataract extraction in a spider monkey (Atelesgeoffroyi). J. Zoo. An. Med. 11, 58-60.

Wilson, M.E. and Trivedi, R.H. 2012. Axial length measurement techniques in pediatric eyes with cataract. Saudi. J. Ophthalmol. 26, 13-17.

Yorston, D. 2001. Intraocular lens (IOL) implants in children. Community. Eye. Health. 14, 57-58. 